\title{
Parameters Tuning via Simplex-Search based Model-Free Optimization for the Steam Generator Level Control
}

\author{
Guan Jian Sheng* and Kong Xiangsong
}

\author{
School of Electrical Engineering and Automation, Xiamen University of Technology, Xiamen, Fujian 361024, China
}

\begin{abstract}
Control performance is critical to a control system. To improve the performance of the steam generator level control system, the control system parameters need to be optimized. Traditional parameters tuning methods, such as trial and error and Design of Experiments etc., are usually experience-based, cumbersome and time-consuming. To address the above inefficiencies, in this paper, the simplex-search based Model-Free Optimization(MFO) has been proposed to search for the optimal control system parameters. The optimized parameters will be gained to maximize the system's control performance. Rather than traditional controller parameter tuning method, this method optimizes the control system by directly using measurements of control performance. An example of the PID parameters tuning for the steam generator level control was illustrated. The efficiency and the effectiveness of the Simplex-search based Model-Free Optimization - based control parameters tuning methodology has been verified through simulation experiments.
\end{abstract}

Keywords: Steam generator level control, parameters tuning, simplex search, model-free optimization (MFO).

\section{INTRODUCTION}

Steam generator (SG) is the principle interface for the exchange of heat between the primary and secondary side in a pressurized water reactor (PWR). It is a dynamic component and plays an important role in a PWR [1]. About 25\% of emergency shutdowns in the nuclear power plants are caused by poor control of the SG water level [2]. Therefore, maintain the steam generator level within allowable limits is critical to the safety and economical operation for a nuclear power plant [3]. To achieve the efficient control of the steam generator level, the control performance of the system should be improved. However, the SG is a nonlinear and complicated industrial process with uncertainties. Thus, to improve the control performance of the steam generator level control system is a challenging task.

The control performance of the steam generator level control system is determined by the process characteristics of the SG, the structure of the control system and the controller parameters. In a nuclear power plant, to a certain steam generator, the process characteristics are fixed yet. And once the design of the control system is finished, the structure of the control system is determined too. Then, the controller parameters are the only left key factors. The controller parameters have a close relationship with the steam generator level control performance. Controller parameters optimization is an important way to improve the performance of the steam generator level control system by searching for the optimal parameters settings [4]. For example, PID parameters tuning is a typical controller parameters optimization problem.
There are three main approaches used to search for the optimal controller settings [5]. The first one is the trial-anderror method. With this method, the engineers tune the parameters by trial experiments on their own experience. They conduct experiments and adjust the controller parameters according to the results, the process will go on until the control performance meets the requirements. This approach are usually time consuming and costly, and the optimality of the settings can not be ensured either.

The second one is based on the design of experiments (DOE) [6, 7]. It uses relatively less number of experiments by well-designed plans, such as Latin Hypercube Sampling (LHS), Taguchi design and Uniform Design etc. This method reduces the costs of the optimization, it is simple and easy for implementation. However, this method depends on a lot of human interventions and usually can only get suboptimal settings.

The third one is the model-based method (MBO). Suppose there is a model which represents the relationship between the control performance and the parameter settings. If the model of the control performance is known, the optimal settings can be obtained through the model-based optimization method. However, the relationship between the controller performance and the parameters is always complicated that the model is usually unavailable. In controller parameters tuning, the formula-based method is widely used by the control engineers. For instance, the Ziegler-Nichols tuning method and the Åström-Hägglund phase margin method. They are typical formula-based method for the PID parameter tuning [8-11]. The formula-based method can be treated as a special case of the model-based method. These methods depend on a formula, which represents the relationship between the control performance and the parameter settings. For this method, the engineers should obtain the process model firstly through transient response experiments or pa- 


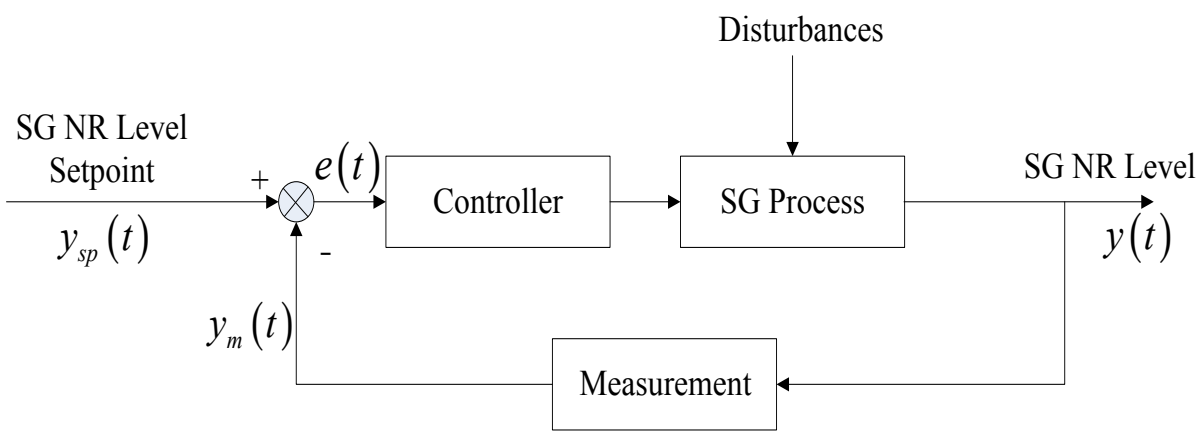

Fig. (1). The structure of the steam generator level control system.

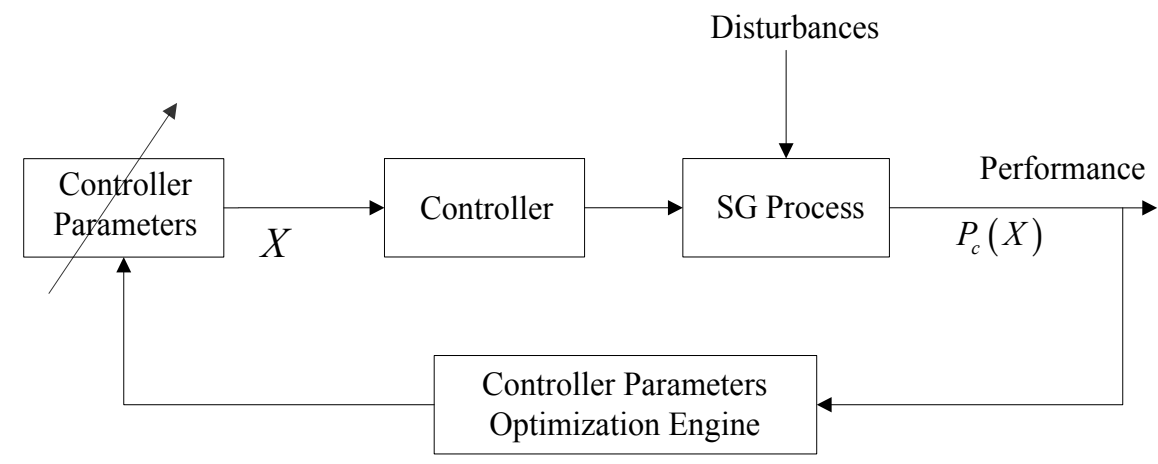

Fig. (2). The schematics of the control parameters optimization.

rameters estimation or frequency response experiments. Then they can obtain the optimal settings according to certain tuning formulae developed based on the model. This method is easy for implementation. However, this method relies on the knowledge of the process. There are three main disadvantages exists. Firstly, the accurate process model is always difficult or unavailable to build. Secondly, the optimal settings obtained by the formula-based method are usually suboptimal settings. Thirdly, the tuning formula has a close relationship with the type of the process model; choosing a suitable formula depends on the engineer's own experience and a perfect knowledge of the process. All these make this method can only give poor results in many cases. For this reason, the application of the traditional modelbased optimization method is limited in controller parameters optimization.

As stated above, all the three main approaches have their shortcomings, they are inefficient in controller parameters optimization. It is necessary to find an efficient method to search for the optimal controller parameters settings. To obtain a novel method to address the shortcomings of the traditional methods, the characteristics of the tuning process should be analyzed. The process of the parameters tuning can be divided into four stages: (1) set a group of parameters; (2) conduct an experiment and measure the control performance; (3) evaluation, then stop the tuning process and go to stage 4 or determine the new group parameters and go back to the first stage; (4) output the optimal settings. Considering the characteristics of the controller parameters, the parameters tuning process just like a batch process. Methods that is efficient in batch process optimization may be suit for the parameters tuning.
Kong etc. has proposed a systematic and efficient modelfree optimization method for the injection molding quality control and optimization $[12,13]$. Using the quality measurements instead of a model, this method can obtain the optimal process conditions efficiently in a limited number of experiments. Injection molding is a typical batch process. The controller parameters optimization problem is similar to the quality control and optimization of injection molding. Due to this, in this project, the simplex-search based MFO has been proposed to the controller parameters optimization of steam generator level control.

\section{PARAMETERS TUNING OF THE STEAM GEN- ERATOR LEVEL CONTROL}

The steam generator level control system is constructed to maintain the steam generator water level at a desired value with allowable bounds. A simplified structure of the steam generator level control system is shown in the following Fig. (1). Although the framework of the control system are always the same, the performance of a certain control system usually differs from each other. To obtain better control performance, the control system should be optimized. Therefore, there is a need for performance improvement of the level control system.

Once the SG process and the structure of the level control system have been determined, the control system's performance is only affected by the parameters of the controller. The schematic of the controller parameters optimization can be seen in Fig. (2). The controller parameters optimization process is to tune the controller parameters and search for the 


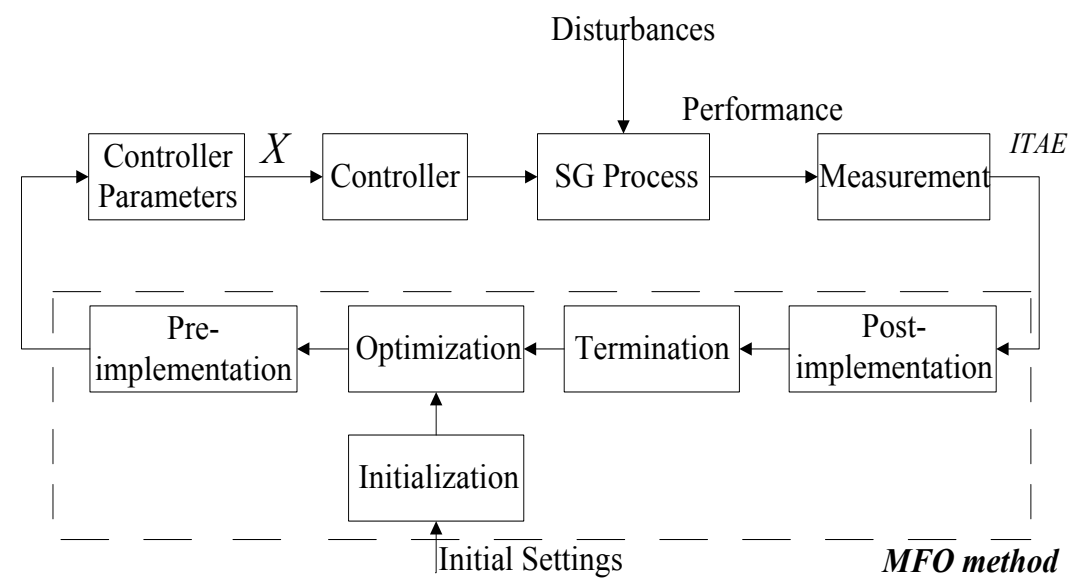

Fig. (3). The schematics of the MFO.

optimal settings. The critical component in the optimization system is the controller parameters optimization engine, which dynamically changes the controller parameters and accomplish the optimization.

Suppose there are a number of $\mathrm{n}$ controller parameters to be optimized. They can be represented by $x_{1}, x_{2}, x_{3}, \cdots, x_{n}$, separately. So, a group of controller parameters settings can be defined as a vector $x=\left[x_{1}, x_{2}, x_{3}, \cdots, x_{n}\right]^{T}$. All the $\mathrm{n}$ parameters affect the controller's performance together. There will be settings whose performances may be better than the others. The controller parameters optimization is to find the optimal settings among them. The mathematical formula of the optimization problem can be expressed as below:

$\max \operatorname{Perf}=f(x)$

s.t. $x \in \Omega$

where $x$ represents the controller parameters settings, $\Omega$ is the feasible region of the settings, Perf is the control performance and it is a function of $x . f(x)$ is the relationship between the control performance and the controller parameters. However, $f(x)$ is usually unavailable or too difficult to obtain.

Feedback is a necessary part of a close-looped control system. To accomplish the optimization, control performance measurement is critical. It is necessary to find a way to evaluate the control performance of the steam generator level control system. For a control system, regulation performance is often expressed in terms of the control error obtained under certain disturbances. Typical control evaluation index can be expressed as below:

$I=\int_{0}^{\infty} t^{n}|e(t)|^{m} d t$

where $I$ represents the control index; and the error is defined as $e(t)=y_{s p}(t)-y(t), y_{s p}(t)$ is the control target trajectory and $y(t)$ is the actual response.
With the above evaluation index, the controller performance optimization problem can be expressed as below:

$$
\begin{aligned}
& \min -\operatorname{Perf} \approx I=\int_{0}^{\infty} t^{n}|e(t)|^{m} d t \\
& \text { s.t. } x \in \Omega
\end{aligned}
$$

The performance is approximately represented by the evaluation index $-I$. In this project, the Integral of Time multiply by Absolute Error (ITAE) index is chosen. The ITAE is an index widely used in control performance evaluation with $n=1, m=1$ in Eqn. (3). When the absolute value of the index gets bigger, the performance of the control system gets worse.

After the problem formulation, the methodology that proposed to resolve the problem will be described in the next section.

\section{SIMPLEX-SEARCH BASED MFO}

\section{A. Model-Free Optimization (MFO)}

Traditional experience-based methods are cumbersome and cannot ensure the optimality of the control performance. Due to the difficulty to obtain the accurate model of the control performance, the model-based optimization method cannot be implemented on the problem efficiently either. It is necessary to develop a more efficient method for the control parameters optimization. Model-free optimization (MFO) can provide a new way to this problem.

MFO is a suitable method for the optimization problem of Eqn. (3) that has the same characteristics of batch process. This method can avoid the shortcomings of the above three traditional methods. This method does not need to build a control performance model. Instead, it uses the directly performance measurements to evaluate the control performance.

The framework of how the MFO works is shown in Fig. (3). It can be seen that the MFO is consisted of several function modules [13]. All the modules are connected with each other and become an integrated methodology. The operation process of the MFO is as follows: at each group of 
controller parameters settings, a transient is conducted on the steam generator process, the response of the steam generator level is recorded and the ITAE index will be calculated. The control performance represents by the ITAE will then delivered to the post-implementation module of the MFO. After the post-implementation, the feedback results will be transferred to the termination module. At that stage, the MFO will judge whether the termination rule is met. If the termination rule is not met, the method will generate the next iteration point and the corresponding settings will be delivered to the controller through the pre-implementation module. This process repeats until the termination rule is finally met.

In this project, the simplex-search based model-free optimization (MFO) has been proposed for the controller parameters optimization. The following subsection will introduce the simplex search algorithm and the simplex-search based MFO.

\section{B. Simplex-Search based MFO}

Simplex search is proposed by Nelder and Nead, it is an efficient algorithm that works well in industrial applications $[14,15]$. The simplex search minimizes a function of $p$ variables by the comparison of function evaluations at the $p+1$ vertices of a general simplex. And the simplex will updates by the replacement of the vertex with the highest value by another vertex. Through this, the simplex will gradually adapts itself to the local landscape and contracts on to the final minimum. A simplex is a general geometric object that is the convex of $p+1$ points in the $p$ dimensional space, $R p$. The algorithm minimizes the function of $\mathrm{p}$ variables depending on the comparison of function values at the $p+1$ vertices of the simplex, followed by the replacement of the vertex with the highest value by another point. Normally, the optimal results can be obtained after a limited number of iterations. This algorithm is effective due to its rapid convergence to the optimal settings.

From the view of model-free optimization, the simplex search is a gradient-free algorithm which uses the function evaluations directly [13]. The gradient-free method is advantageous in that it does not require any gradient calculation, which may entail numerous experiments in the MFO, especially in cases where the optimization problem has a large dimension. Due to these reasons, the simplex search can be incorporated in the MFO too. And then, the simplex-search based MFO is formulated.

Simplex search updates the simplex to search for improvement. It can be divided to the following steps:

\section{a) Initializing}

Formulate the initial simplex; the simplex vertex number is one larger than dimension of optimization problem. Every elements of the initial guess $x_{0}$ are normalized to the same range, $[0,100]$. Construct the initial simplex with the normalized initial point $\bar{x}_{0}$ using a sequentially perturbation method. Through this method, we will perturb every components of $\bar{x}_{0}$ with a perturbation ratio $\tau(\tau \in(0,50 \%])$, and record the function evaluation value at each vertex. After that, the per- turbed vector and the original initial point construct a simplex with $\mathrm{p}+1$ vertices, $\left\{v_{1}, v_{2}, \cdots, v_{p+1}\right\}$.

b) Ranking

To label the best $\left(v_{1}\right)$, the worst $\left(v_{p+1}\right)$ and the next-toworst $\left(v_{p}\right)$ vertices in the current simplex. The evaluation standard is by the function value of each vertex. Assuming that we are seeking to minimize the process output, the worst vertex $v_{p+1}$ is the one that with the highest function value.

c) Reflection

A reflection is done as $v_{r e f}=(1+\alpha) v_{m}-\alpha v_{p+1}$.

where $v_{m}=\left(\sum_{i=1}^{p} v_{i}\right) / p$

$v_{m}$ is the center of all the vertex except the worst one; $\alpha$ is the reflection coefficient. Denote the function evaluation of objective as $-\operatorname{Perf}(x)$. If $-\operatorname{Perf}\left(v_{r e f}\right) \leq-\operatorname{Perf}\left(v_{1}\right)$, an expansion operation is activated, go to step (d); else if $-\operatorname{Perf}\left(v_{\text {ref }}\right)>-\operatorname{Perf}\left(v_{1}\right)$, a contraction operation is adopted, go to step (e); else, $v_{r e f}$ is accepted to replace $v_{p+1}$, go to step (f).

d) Expansion

An expansion operation is done as

$v_{\text {exp }}=(1-\gamma) v_{m}+\gamma v_{r e f}$

where $\gamma$ is the expansion coefficient. If $-\operatorname{Perf}\left(v_{\text {exp }}\right) \leq-\operatorname{Perf}\left(v_{\text {ref }}\right), v_{\text {exp }}$ will be adopted to replace the worst one $v_{p+1}$, otherwise $v_{p+1}=v_{r e f}$; then go to step (e).

e) Contraction

In this operation, the contraction point is defined as:

$v_{c t}=(1-\beta) v_{m}+\beta v_{\max r e f}$

where $\beta$ is the contraction coefficient; $v_{\text {max ref }}$ is an undetermined variable depending on different conditions. When the function value $-\operatorname{Perf}\left(v_{r e f}\right)$ of $v_{r e f}$ is greater than that of the maximum point $v_{p+1}$, an inside contraction will be implemented and $v_{\text {max ref }}$ is set to $v_{p+1}$; else an outside contraction will be adopted and $v_{\max r e f}=v_{r e f}$.

After the contraction, the function values of $v_{c t}$ and $v_{\max / r e f}$ are compared. If $y(t)=C x(t)+D u(t)$, the contraction point is accepted to replace $v_{p+1}$; otherwise there is no performance improvement, a shrink operation is adopted, go to step (f); 
Table 1. Parameters of a typical steam generator.

\begin{tabular}{|c|c|c|c|c|c|}
\hline$P(\%)$ & 5 & 15 & 30 & 50 & 100 \\
\hline$\overline{G_{1}}$ & 0.058 & 0.058 & 0.058 & 0.058 & 0.058 \\
\hline$G_{2}(P)$ & 9.63 & 4.46 & 1.83 & 1.05 & 0.47 \\
\hline$G_{3}(P)$ & 0.181 & 0.226 & 0.310 & 0.215 & 0.105 \\
\hline$\tau_{1}(P)$ & 41.9 & 26.3 & 43.4 & 34.8 & 28.6 \\
\hline$\tau_{2}(P)$ & 48.4 & 21.5 & 4.5 & 3.6 & 3.4 \\
\hline$T(P)$ & 119.6 & 60.5 & 17.7 & 14.2 & 11.7 \\
\hline$Q_{w}(\mathrm{~kg} / \mathrm{s})$ & 57.4 & 180.8 & 381.7 & 660 & 1435 \\
\hline
\end{tabular}

f) Shrink

A shrink operation is done as

$$
v_{i}=\delta v_{i}+(1-\delta) v_{1}, i=2, \cdots, p .
$$

Each vertex except the minimum one is replaced by a new vertex according to the above equation;

\section{g) New simplex}

A new simplex is constructed. All the new vertices will be treated as $\bar{x}_{k}$ and be evaluated by online experiments.

The simplex-search based MFO is constructed, and it will be implemented on the parameters tuning problem in this project.

\section{CASE STUDY}

\section{A. Steam Generator Model}

Steam generator of a nuclear power plant is a complex and nonlinear system. In this study, a simplified steam generator level model, which was proposed by E. Irving [2], has been used to simulate the steam generator level process. This model has been widely used for the simulation and control of the steam generator. The transfer function of the model is as follows:

$$
\begin{aligned}
& Y(s)=\left(\frac{G_{1}}{s}-\frac{G_{2}}{1+\tau_{2 \mathrm{~S}}}\right)\left[Q_{e}(s)-Q_{v}(s)\right]+ \\
& \frac{G_{3} S}{\tau_{1}^{-2}+4 \pi^{2} T^{-2}+2 \tau_{1}^{-1} s+s^{2}} Q_{e}(s)
\end{aligned}
$$

where $\mathrm{Y}(\mathrm{s})$ is the output of the model, which represents the narrow range water level of the steam generator. The system has two inputs. The first one is the feed water rate, which is represents by $\mathrm{Qe}(\mathrm{s})$; the second one is the steam rate represents by $\mathrm{Qv}(\mathrm{s}) . \mathrm{G} 1, \mathrm{G} 2, \mathrm{G} 3, \tau_{1}, \tau_{2}$ and $\mathrm{T}$ are parameters of the model. G1 is the magnitude of the mass capacity effects, G2 is the magnitude of the swell and shrink phenomena, and G3 is the magnitude of the mechanical oscillation. $\tau_{1}, \tau_{2}$ are the damping time constants. $\mathrm{T}$ is the period of the mechanical oscillation. All these parameters are highly related with the power $\mathrm{P}$ of the nuclear power plant, they can be treated as the function of the power. When the power level $\mathrm{P}$ varies, the parameters usually changes. The model parameters at different power levels have been identified from experimental data by E. Irving, and the identified parameters are given in Table $\mathbf{1}$.

In order to simulate the steam generator level process with Simulink, the state space equations of the steam generator has been derived from Eqn. (9):

$$
\begin{aligned}
& x(t)=A(p) x(t)+B(p) u(t) \\
& y(t)=C x(t)+D u(t)
\end{aligned}
$$

where $u(t)$ is a $2 \times 1$ vector, $\left[u_{1}(t), u_{2}(t)\right]^{T} ; \mathrm{u} 1(\mathrm{t})$ represents the feedwater flow and $\mathrm{u} 2(\mathrm{t})$ represents the steam flow. The matrix is as follows:

$$
\begin{aligned}
& A(p)=\left[\begin{array}{cccc}
0 & 0 & 0 & 0 \\
0 & \frac{-1}{\tau_{2}} & 0 & 0 \\
0 & 0 & -2 *\left(\frac{1}{\tau_{1}}\right) & 1 \\
0 & 0 & -\left(\frac{1}{\tau_{1}}\right)^{2}-4 * \pi^{2} * \tau \frac{1}{T} \tau^{2} & 0
\end{array}\right] \\
& B(p)=\left[\begin{array}{cc}
G_{1} & -G_{1} \\
\frac{-G_{2}}{\tau_{2}} & \frac{G_{2}}{\tau_{2}} \\
G_{3} & 0 \\
0 & 0
\end{array}\right]
\end{aligned}
$$

$C(p)=\left[\begin{array}{llll}1 & 1 & 1 & 0\end{array}\right]$

$D(p)=\left[\begin{array}{ll}0 & 0\end{array}\right]$

The S-function based steam generator level model is constructed according to the above equivalent state space model. 
OVERVIEW of SG Model

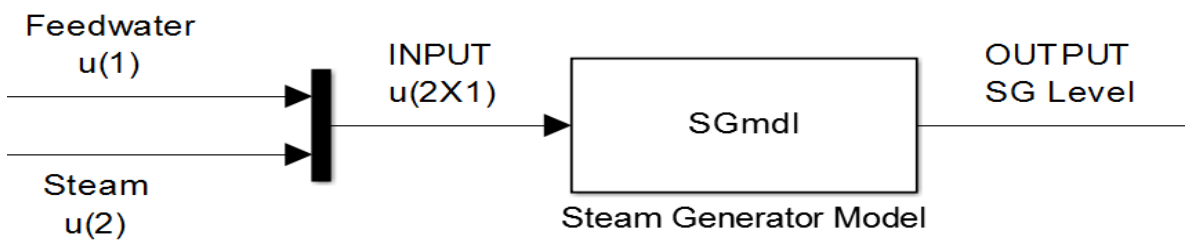

Fig. (4). Encapsulated Steam Generator Model and its external interfaces.

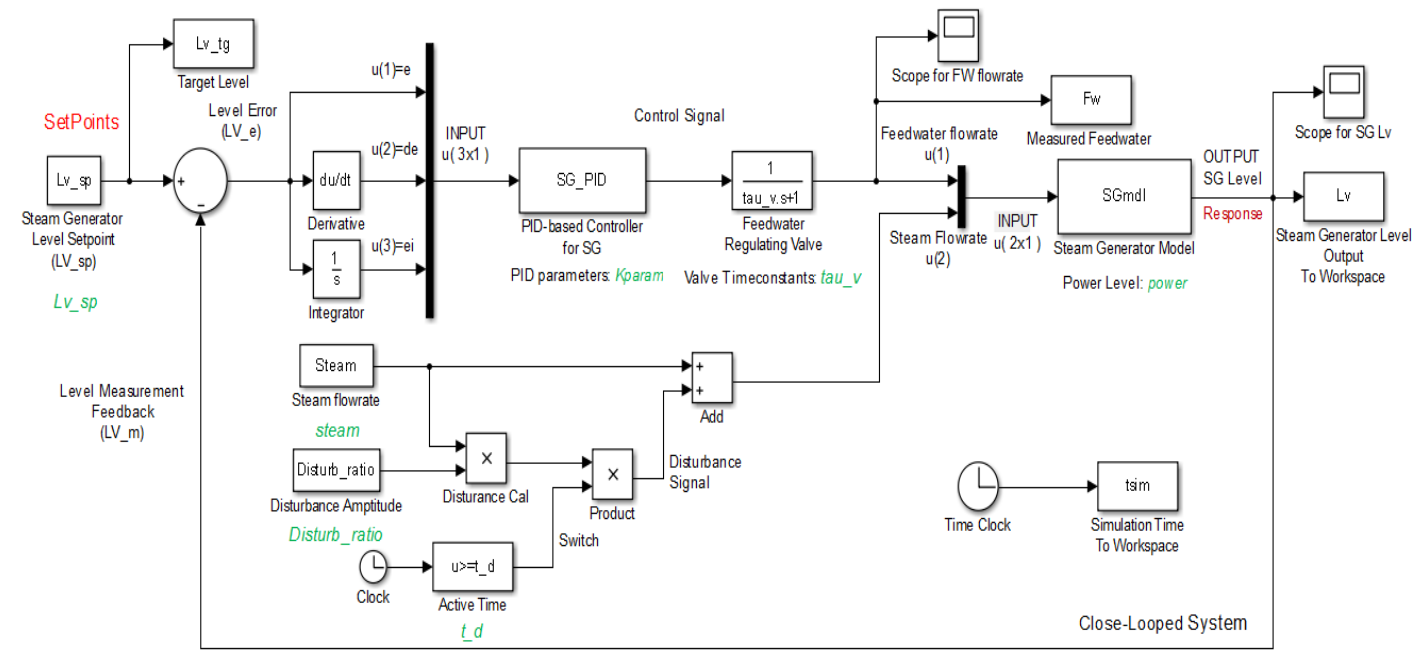

Fig. (5). Steam generator level control system.

The Fig. (4) shows the interfaces of the encapsulated model build in Simulink. The two inputs of the model are the feedwater flow and the steam flow and the output of the model is the level of the steam generator.

Based on the above model, a steam generator level control system built by the Simulink has been constructed. The level control system is shown below in Fig. (5).

As the process and the structure of the control system have been determined, the performance of the level control system will be mainly determined by the parameters of the control system. As the single-impulse PID controller is chosen, the most significant parameters are the PID parameters. The P-I-D parameters are critical to the control performance of the system. Generally speaking, the proportional gain is used to control how much the proportional part of the algorithm will affect the output value. The integral reset time determinates how much time will be required for the error to be integrated out by the integral part of the algorithm. Derivative gain is used to control how much the derivative part of the algorithm will affect the output value. The derivative rate constant is used to control how fast the derivative part of the algorithm will decay to zero.

The PID rule is as follows:

$k_{P}+\frac{k_{I}}{s}+k_{D} \cdot s$ where $k_{P}$ represents the proportion gain, $k_{I}$ represents the integral gain, $k_{D}$ represents the derivative gain. They can be replaced by $x_{1}, x_{2}, x_{3}$. The PID parameters of the control system then be defined as $x=\left[x_{1}, x_{2}, x_{3}\right]^{T}$. The feasible region of the PID parameters in this project are shown in Table 2. To optimize the performance of the control system, the three parameters settings should be adjusted. Parameters Tuning process is a process to find the optimum parameters settings in the fixed feasible region.

\section{B. Results and Discussions}

During the parameters tuning process, a single simulation experiment should be conducted for each PID parameters settings. The corresponding performance at that settings will be calculated according to the response of the experiment. For a whole optimization process, a typical process condition is adopted. During the optimization process, the initial conditions at each experiment with different PID parameters are always the same.

In the project, to verify the effectiveness of the simplexsearch based MFO on parameters tuning, different process conditions with different initial points are tested by simulation experiments. 
Table 2. Feasible region of the PID parameters.

\begin{tabular}{|c|c|c|c|}
\hline Parameters & $x_{1}$ & $x_{2}$ & $x_{3}$ \\
\hline \hline Low Limits & 0 & 0 & 0 \\
\hline Upper Limits & 6 & 0.5 & 0.5 \\
\hline
\end{tabular}

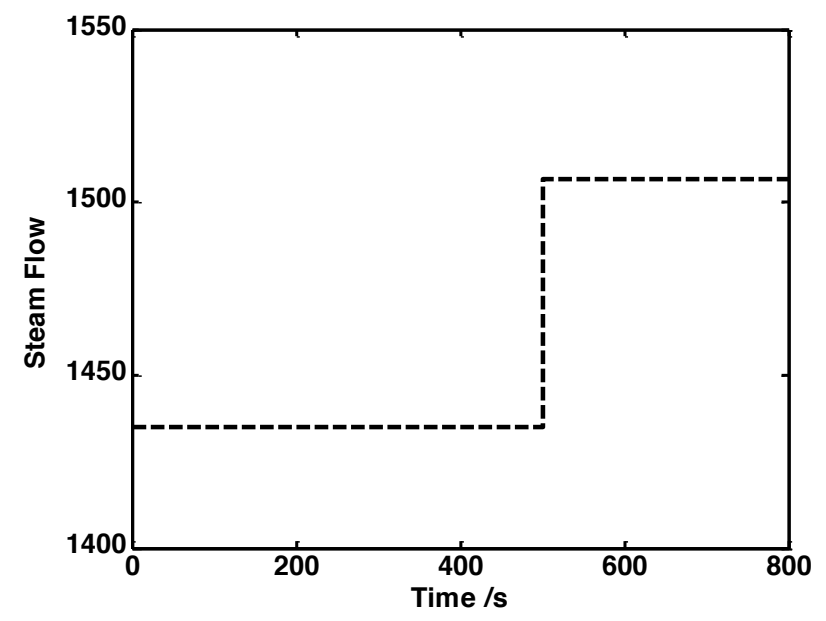

Fig. (6). Trajectory of the steam flow.

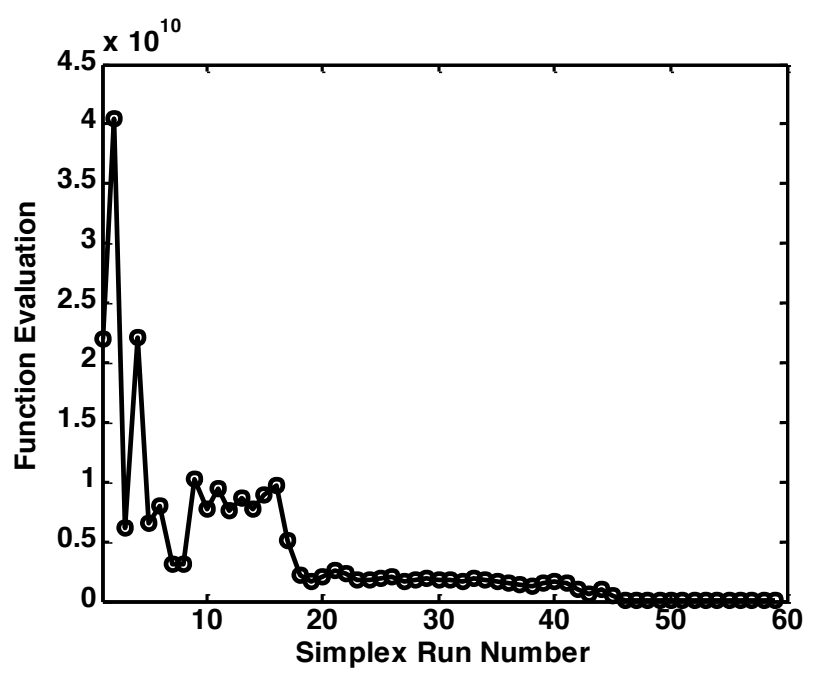

Fig. (7). Trajectory of the simplex-search based MFO Iteration points.

1. Step change of $5 \%$ of Steam flow at full power:

The nuclear power plant is in operation under $100 \%$ rated power. For the model simulation, $0 \sim 500$ s are the model initialization stage. The simulation model will fallen into a stable status in 500 seconds. When the plant is surely at the steady status, at the 500 seconds, the balance of the steam generator level will be broken. A step change of 5\% in steam flow will occur, the steam generator level control system go into the transient response process. The trajectory of the steam flow is as follows. At the $500 \mathrm{sec}$, a perturbation of 5\% step change is occurred Fig. (6).
When the transient occurs, the steam generator level control system will try to maintain the level at the setpoints through regulation of the feedwater flow. The performance of the control system will mainly depends on the parameters settings of the PID controller. In this project, the simplexsearch based MFO is implemented to accomplish the controller parameters tuning.

Suppose an initial guess $\mathrm{x} 0=[4,0.3,0.3] \mathrm{T}$ is selected by random. With the simplex-search based MFO, the parameters tuning is finished successfully in limits iterations. The optimization trajectory of the tuning process can be seen in Fig. (7). It can be seen during the parameters tuning process, 


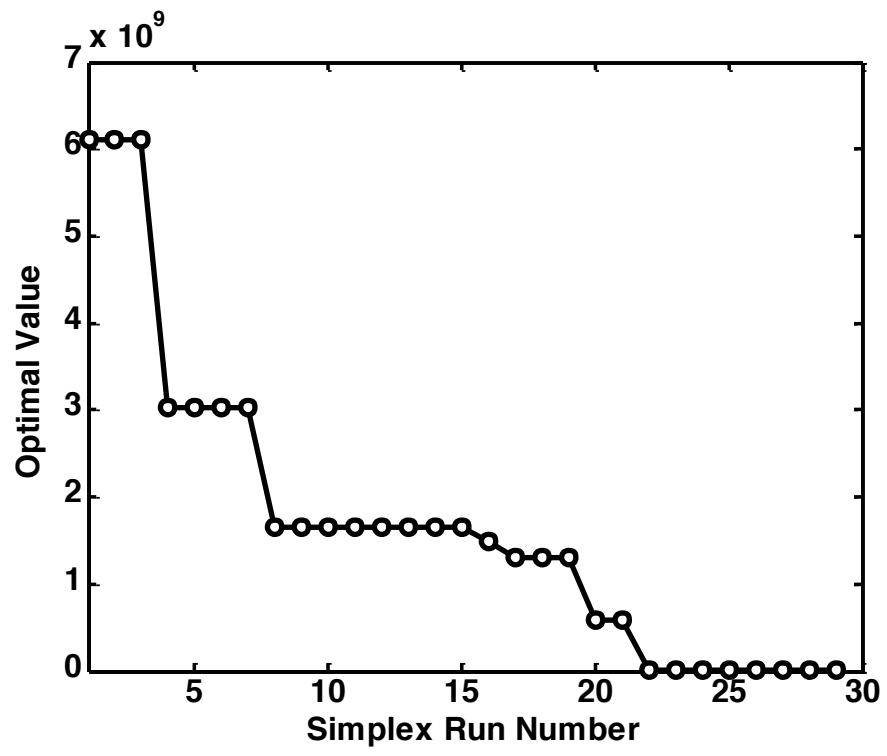

Fig. (8). Trajectory of the optimum points under the simplex-search based MFO.

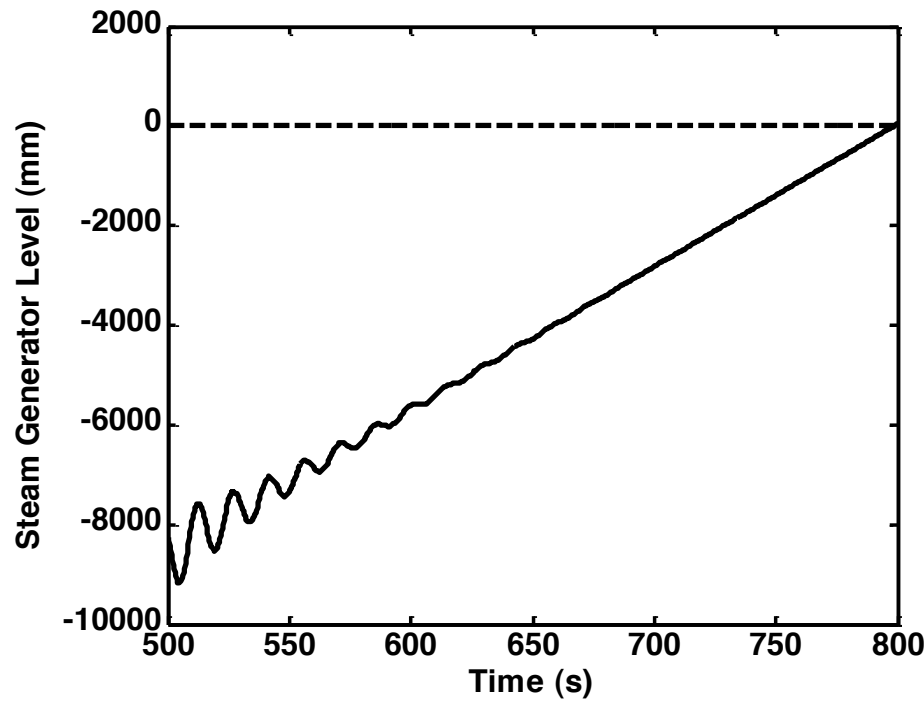

Fig. (9). Steam Generator Level Response at the initial guess before optimization

the performance of the control system greatly increased in limits iterations. To monitor the optimum settings' progress, the trajectory of the optimum points under the simplexsearch based MFO has been recorded. It can be seen in Fig. (8). The trajectory shows the improvement under the simplex-search based MFO in a limit iterations. Finally, the optimized point got by the simplex-search based MFO is $\mathrm{xopt}=[2.16,0.02,0.49] \mathrm{T}$. To show the performance differences between the initial guess $\mathrm{x} 0$ and the optimized point xopt, the response under the two different settings are shown is Fig. (9 and 10). From the figures, we can clearly see the greatly improvement from $\mathrm{x} 0$ to xopt. The effectiveness of the simplex-search based MFO is clearly demonstrated.
To verify the effectiveness of the simplex-search based MFO at different starting points, another different initial point has been tested. The initial point is also selected randomly as $\mathrm{x} 0=[3,0.25,0.25] \mathrm{T}$. The simplex-search based MFO is carried out. It can be seen just as the first demonstration. The PID parameters are tuned iteratively. And during the process, the control performance of the control system is gradually improved. Figs. $(\mathbf{1 1}, \mathbf{1 2})$ shown the process of improvement. Finally, the optimized point got by the simplexsearch based MFO is $\mathrm{xopt}=[2.3,0.016,0.4] \mathrm{T}$. The different control performance at $\mathrm{x} 0$ and xopt can be clearly seen from Fig. $(13,14)$.

2. Step change of $10 \%$ of Steam flow at $50 \%$ rated power: 


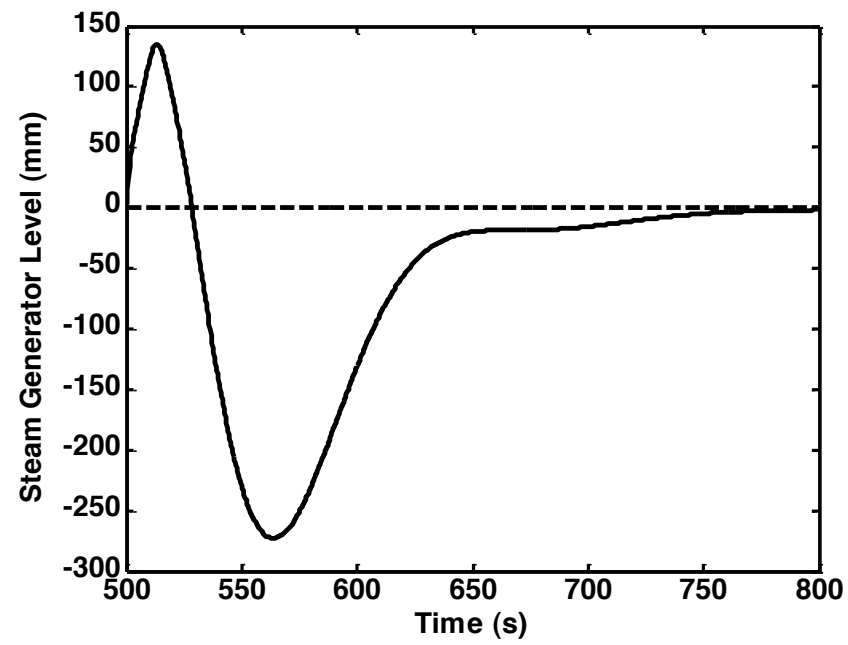

Fig. (10). Steam Generator Level Response at the optimized point after optimization.

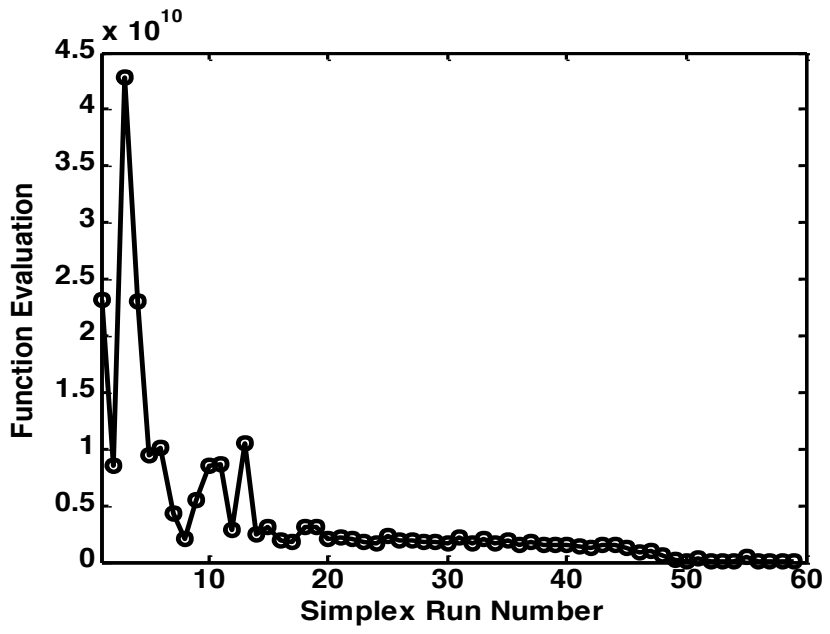

Fig. (11). Trajectory of the simplex-search based MFO Iteration points.

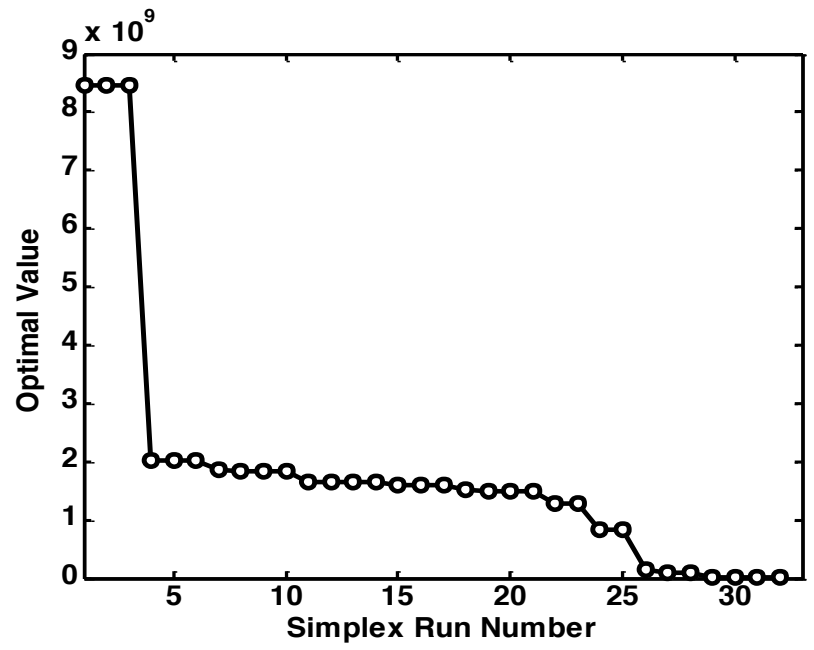

Fig. (12). Trajectory of the optimum points under the simplex-search based MFO. 


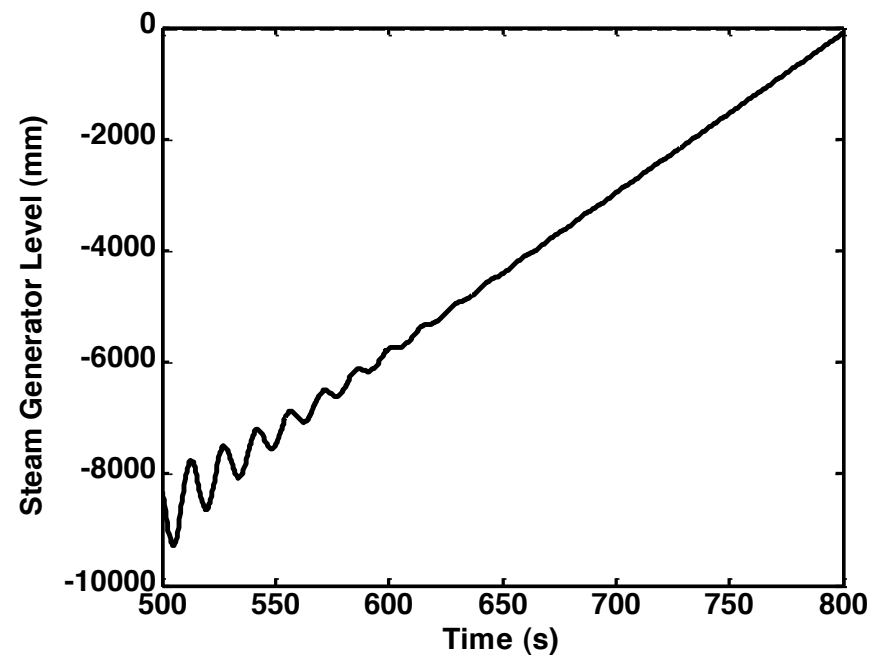

Fig. (13). Steam Generator Level Response at the initial guess before optimization.

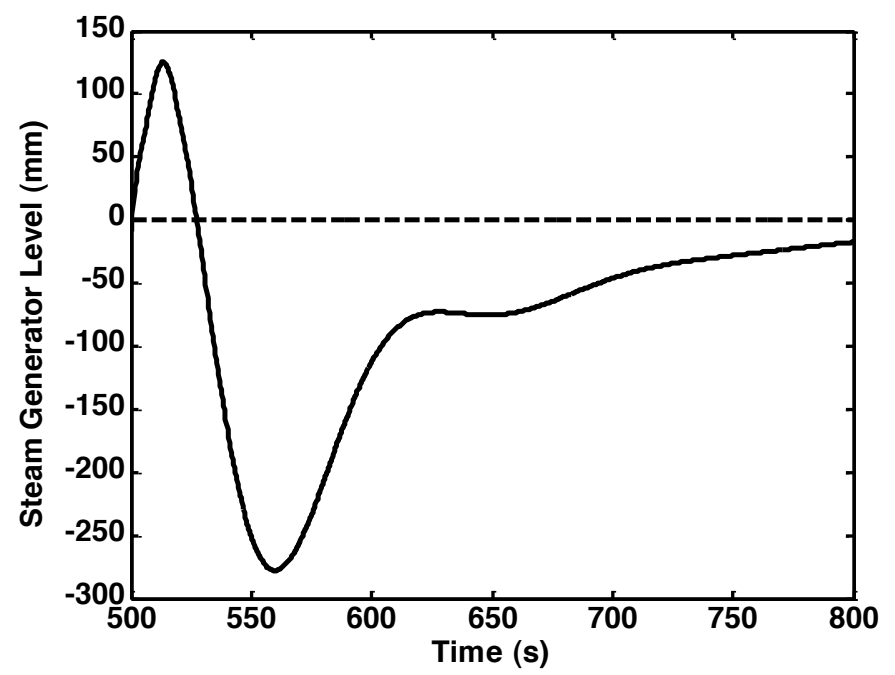

Fig. (14). Steam Generator Level Response at the optimized point after optimization.

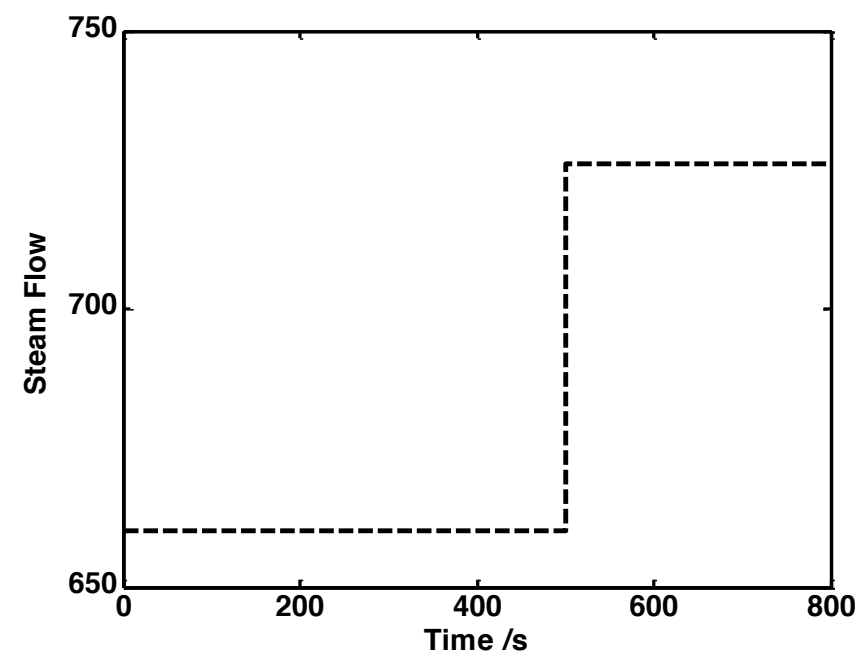

Fig. (15). Trajectory of the steam flow. 


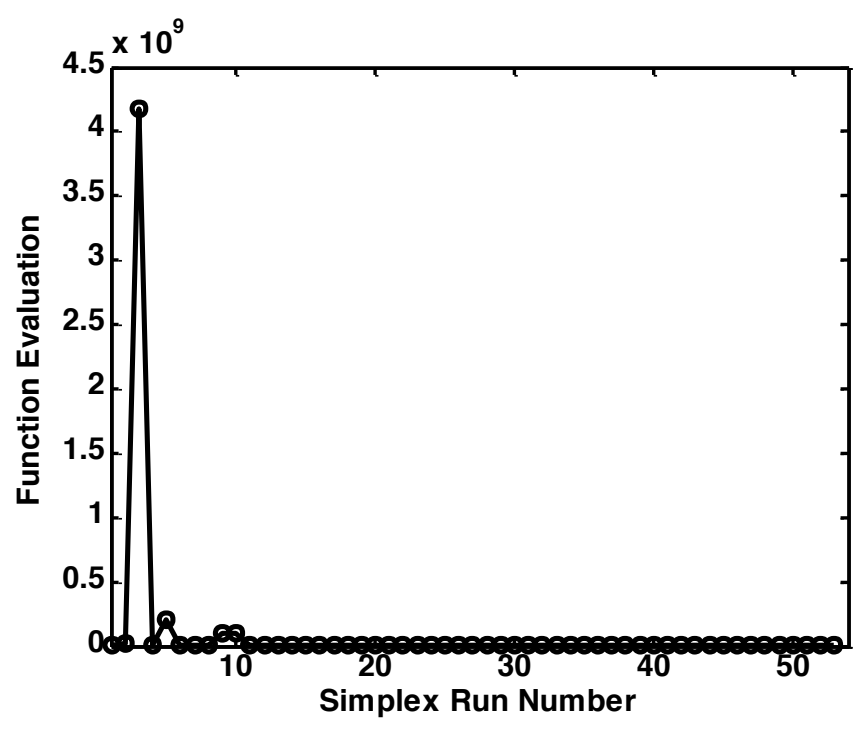

Fig. (16). Trajectory of the simplex-search based MFO Iteration points.

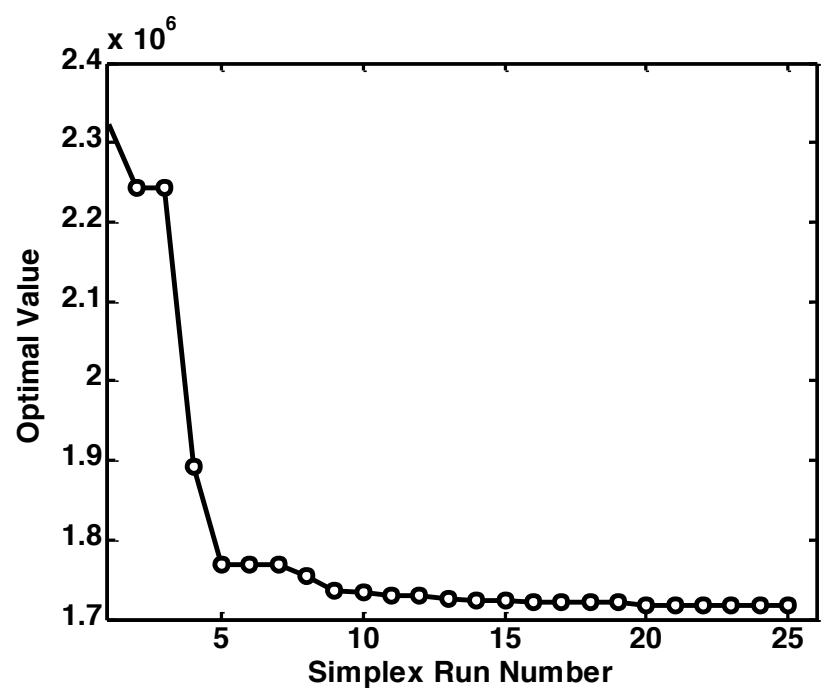

Fig. (17). Trajectory of the optimum points under the simplex-search based MFO.

To test the effectiveness at different operation conditions, another operation condition is selected and simulated. Suppose the nuclear power plant is now in operation under $50 \%$ rated power. For the model simulation, $0 \sim 500 \mathrm{~s}$ are the model initialization stage. The simulation model will fallen into a stable status in 500 seconds. When the plant is surely at the steady status, at the 500 seconds, the balance of the steam generator level will be broken. A step change of $10 \%$ in steam flow will occur, the steam generator level control system go into the transient response process. The trajectory of the steam flow is as follows Fig. (15).

As before, an initial guess $\mathrm{x} 0=[4,0.1,0.2] \mathrm{T}$ is selected by random. With the simplex-search based MFO, the parameters tuning is finished successfully in limits iterations. The optimization trajectory of the tuning process can be seen in Fig. (16). It can be seen during the parameters tuning process, the performance of the control system greatly increased in limits iterations. To monitor the optimum settings' progress, the trajectory of the optimum points under the simplexsearch based MFO has been recorded. Fig. (17) shows the trajectory of the optimum vertex in each simplex. The trajectory shows the improvement under the simplex-search based MFO in a limit iterations. Finally, the optimized point got by the simplex-search based MFO is xopt $=[4.03,0.06,0.31] \mathrm{T}$. To show the performance differences between the initial guess $\mathrm{x} 0$ and the optimized point xopt, the response under the two different settings are shown is Fig. (18 and 19). From the figures, the greatly improvement from $\mathrm{x} 0$ to $\mathrm{xopt}$ are shown. The effectiveness of the simplex-search based MFO is clearly demonstrated.

\section{CONCLUSION}

Parameters tuning of the steam generator level control has been converted to a nonlinear optimization problem. Considering the characteristics of the parameters tuning, the 


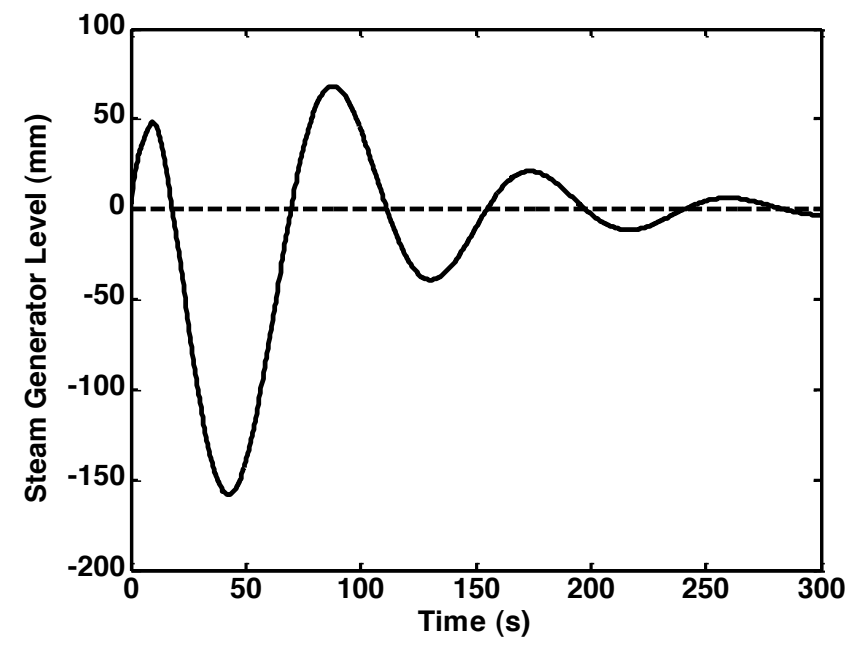

Fig. (18). Steam Generator Level Response at the initial guess before optimization.

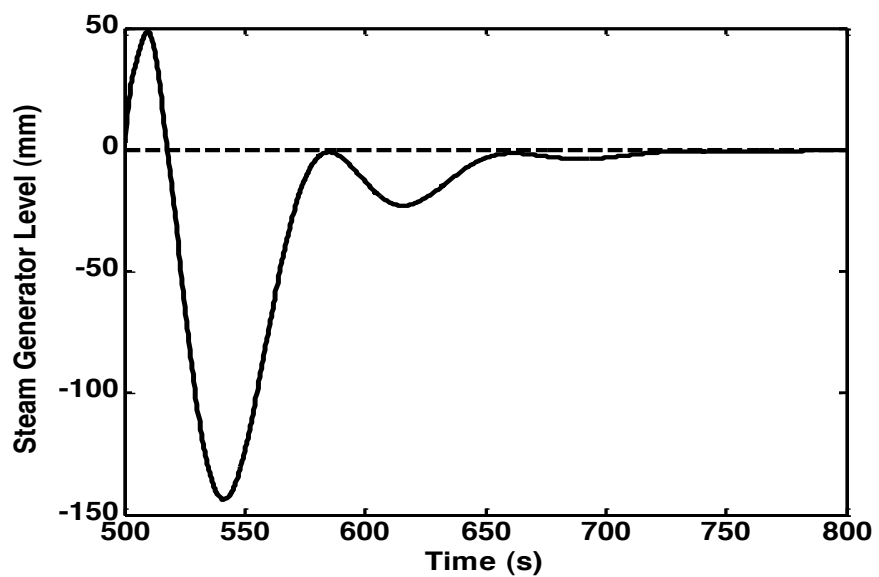

Fig. (19). Steam Generator Level Response at the optimized point after optimization.

tuning process is similar to the batch process. Model-free optimization that has high efficiency on the batch process can suitable for.

To improve the parameters tuning efficiency and reduce the costs that are usually expensive, a novel method of the simplex-search based model-free optimization was proposed to apply on the controller parameters optimization of steam generator level control. The simulation experiments show that this method is efficient in controller parameters optimization. It can quickly converge to the optimal parameters settings. This method can be extended to other controller parameters optimization problems in process control of a nuclear power plant.

\section{CONFLICT OF INTEREST}

The authors confirm that this article content has no conflicts of interest.

\section{ACKNOWLEDGEMENTS}

This work was supported by the Xiamen University of Technology High Level Talents Projects of YKJ13034R and
Supported by A Class Scientific Research Fund of Fujian Provincial Education Department (JA12246).

\section{REFERENCES}

[1] J.J. Sohn, "A steam generator model identification and robust H-inf controller design with v-gap metric for a feedwater control system", Annals of Nuclear Energy, vol. 37, pp. 180-195, 2010.

[2] E. Irving, C. Miossec and J. Tassart, "Towards efficient full automatic operation of the PWR steam generator with water level adaptive control", Boiler Dynamics and Control in Nuclear Power Stations, pp. 309-329, 1980.

[3] M.V. Kothare, "Level control in the steam generator of a nuclear power plant", IEEE Transactions on Control Systems Technology, vol. 8 , no. 1 , pp. 55-69, 2000.

[4] W. Tan, "Water level control for a nuclear steam generator", $\mathrm{Nu}$ clear Engineering and Design, vol. 241, pp. 1873-1880, 2011.

[5] X. Kong and Z. Ma, "A novel method for controllers parameters optimization of steam generator level control". In the Proceedings of the 21st International Conference on Nuclear Engineering, 2013.

[6] C.F.J. Kuo and T.L. Su, "Optimization of injection molding processing parameters for LCD light-guide plates", Journal of Materials Engineering and Performance, vol. 16, no. 5, pp. 539-548, 2007.

[7] G. E. P. Box and K.B. Wilson, "On the Experimental Attainment of Optimum Conditions", Journal of the Royal Statistical Society Series B-Statistical Methodology, vol. 13, pp. 1-45, 1951. 
[8] M. Zhuang and D.P. Atherton, "Automatic tuning of optimum PID controllers", Control Theory and Applications, IEE PROCEEDINGS-D, vol. 140, no. 3, pp. 216-224, 1993.

[9] W. Wei and C. Tianyou, "A survey of advanced PID parameter tuning methods", Acta Automatica Sinica, vol. 26, pp. 347-355, 2000.

[10] K. J. Åström, "The future of PID control", Control Engineering Practice, vol. 9, pp. 1163-1175, 2001.

[11] Prabhat Agrawal, "Tuning Proportional-Integral-Derivative controllers using achievable performance indices", Industrial \& Engineering Chemistry Research, vol. 42, pp. 5576-5582, 2003.

[12] X. Kong, S. Zhu, Y. Yang, Z. Shao, X. Chen, and L. Zhu, "Quality optimization of injection-molded plastic lenses via model free op- timization method", In SPE Annual Technical Conference (AN$T E C)$. Orlando, USA, 2012.

[13] X. Kong, Y. Yang, X. Chen and Z. Shao, "Quality control via model-free optimization for a type of batch process with a short cycle time and low operational cost", Industrial \& Engineering Chemistry Research, vol. 50, no. 5, pp. 2994-3003, 2011.

[14] J.A. Nelderand and R. Mead, "A simplex-method for function minimization", Computer Journal, vol. 7, no. 4, pp. 308-313, 1965.

[15] X. Chen, L. Zhang and X. Kong, "Automatic velocity profile determination for uniform filling in injection molding", Polymer Engineering \& Science, vol. 50, no. 7, pp. 1358-1371, 2010.

Received: September 22, 2014

(c) Sheng and Xiansong; Licensee Bentham Open.

This is an open access article licensed under the terms of the Creative Commons Attribution Non-Commercial License (http://creativecommons.org/licenses/by-nc/3.0/) which permits unrestricted, non-commercial use, distribution and reproduction in any medium, provided the work is properly cited. 